\title{
Diagnostic Difficulties and Management of Childhood Lymphoma: A Case Report
}

\author{
M. Tien Yu Song, A. Ralitera, C. U. Rasolofoson, J. S. Bemora, E. C. Rasolonjatovo, \\ W. Ratovondrainy, R. Mamiarisoa, and C. Andriamamonjy
}

\section{ABSTRACT}

Primary dural lymphoma is a rare pathology, it is part of the malignant lymphoma of the central nervous system and it can reach the extradural or subdural space. We report a case of a little boy of three years and two months admitted to the Department of Neurosurgery CHU JRA for multiple swellings in the scalp associated with an altered general condition. The CT scan revealed a multiple extra-axial expansive process with retro-orbital extension responsible for an important exophthalmos as well as at the scalp level. Anatomopathological examination of the dura mater biopsy revealed a malignant non-Hodgkin's small cell lymphoma. The patient underwent chemotherapy of 05 sessions and the evolution was marked by the persistence of the symptoms. The post chemotherapy brain scan showed considerable extension of the lesions. The next step would be radiotherapy.

Keywords: Chemotherapy, Dural lymphoma, Radiotherapy, Small cell non-Hodgkin's lymphoma.

\section{INTRODUCTION}

Malignant non-Hodgkin's lymphomas are hematologic malignancies characterized by abnormal monoclonal proliferation of lymphoid cells that include $\mathrm{B}, \mathrm{T}$ and $\mathrm{NK}$ lymphocytes at different degrees of maturation. They are the most frequent hematological diseases and are classified as the fifth most frequent cancer in the world [1], [2]. Their incidence is increasing with currently more than 8000 cases per year in France and the United States [3]. This incidence has doubled in twenty years. In Madagascar, 35.82\% of hematological malignancies are non-Hodgkin's malignant lymphomas [1]. However, their localization in the dura mater is rare and even exceptional [4]. Our objective is to report a rare case of non-Hodgkin's lymphoma localization which was dural localization.

\section{OBSERVATION}

This was a little boy of three years and two months old,
Published Online: August 11, 2021

ISSN: $2736-5476$

DOI: $10.24018 /$ ejclinicmed.2021.2.4.70

\section{Tien Yu Song}

Department of Neurosurgery CHU JRA

University of Antananarivo, Madagascar.

(e-mail: miraulletienyusong ${ }^{\circledR}$ hotmail.com)

A. Ralitera

Department of Neurosurgery CHU JRA

University of Antananarivo, Madagascar.

C. U. Rasolofoson

Department of resuscitation anesthesia

Andohatapenaka University of

Antananarivo, Madagascar.

J. S. Bemora

Department of Neurosurgery CHU JRA University of Antananarivo, Madagascar.

E. C. Rasolonjatovo

Department of Neurosurgery CHU JRA

University of Antananarivo, Madagascar.

W. Ratovondrainy

Department of Neurosurgery CENHOSOA

Antananarivo Madagascar.

R. Mamiarisoa

Department of Neurosurgery CHU

Tambohobe Fianarantsoa Madagascar.

C. Andriamammonjy

Department of Neurosurgery CHU JRA

University of Antananarivo, Madagascar.

*Corresponding Author with no particular history, admitted to the Neurosurgery Department of the CHUJRA for multiple swellings in the scalp (Fig. 1), which appeared progressively over about two months, associated with a neurological symptom such as tingling and pain in the lower limbs.

The child was conscious with a Glasgow score of 15/15, with an altered general condition. On physical examination, multiple swellings were found on the scalp, firm, painless, with healthy skin opposite (Fig. 1). There was also a slight exophthalmos of the left eye (Fig. 2), non-pulsatile, nonreducible. He had no organomegaly or adenopathy, and the other systems were apparently normal. Biological examinations revealed bicytopenia with normocytic normochromic anemia at $69 \mathrm{~g} / \mathrm{L}$ and severe thrombocytopenia at $42 \mathrm{G} / \mathrm{L}$, very high lactate dehydrogenase (LDH) at $1243 \mathrm{IU} / \mathrm{L}$ and ferritinemia at $910 \mathrm{ng} / \mathrm{ml}$. 


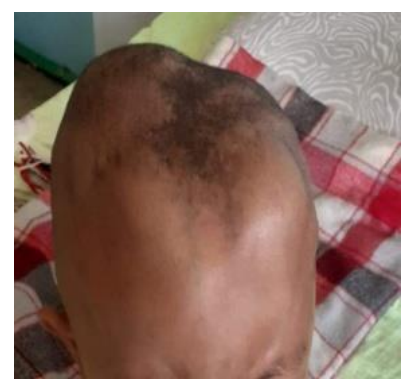

Fig. 1. Multiple swellings were found on the scalp.

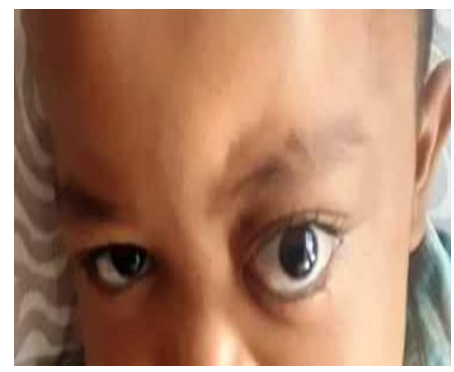

Fig. 2. Exophthalmos of the left eye.
The brain scan showed a spontaneous intracranial hyperdensity of extra-axial bilateral fronto-parietal appearance, $14 \mathrm{~mm}$ thick intracranially and an extra-cranial development of $16 \mathrm{~mm}$ thick right fronto-parietal (Fig. 3). There was also hyperdensity in the right retro-orbital area (Fig. 4).

A biopsy of the scalp swellings was performed for pathological examination (Fig. 5) and revealed a malignant small cell non-Hodgkin's lymphoma. Immunohistochemistry was not available on site.

The patient received repeated blood transfusions and chemotherapy alone with five sessions spaced 21 days apart. The evolution was marked by the absence of regression of symptoms. After the five sessions of chemotherapy, there was an increase in the volume of the swellings (Fig. 6) and the occurrence of the alteration of the general state and the control cerebral CT scan showed an increase in the size of the extra-axial tissue expansive process to $24 \mathrm{~mm}$ in thickness in the left posterior parietal versus $14 \mathrm{~mm}$ before chemotherapy and $35 \mathrm{~mm}$ in the right posterior parietal with a mass effect on the cerebral parenchyma He also demonstrated diffuse osteolytic lesions of the skull creating a "grass fire" image with extension to the scalp and meninges (Fig. 7) and the persistence of a right retro-orbital expansive process responsible for a grade I exophthalmos. Further management would be radiotherapy in collaboration with the medical oncology department.

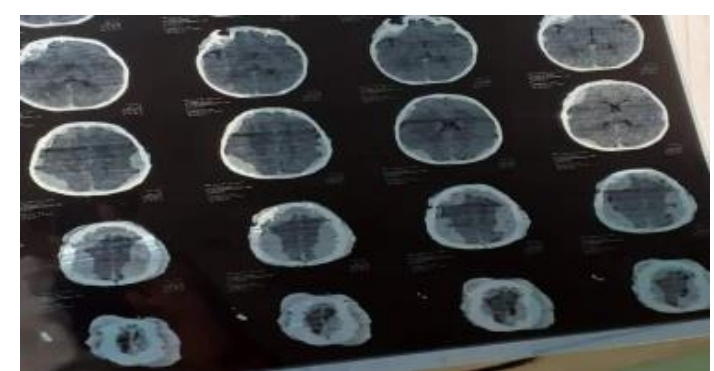

Fig. 3. The brain scan showed a spontaneous intracranial hyperdensity of extra-axial bilateral fronto-parietal appearance.

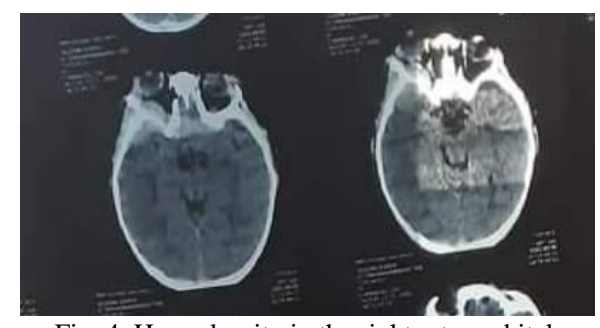

Fig. 4. Hyperdensity in the right retro-orbital.

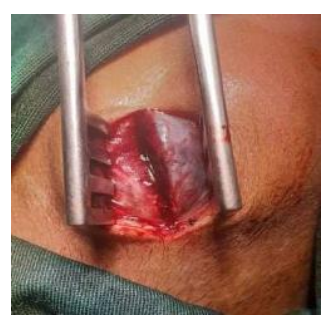

Fig. 5. Biopsy of the scalp swellings.

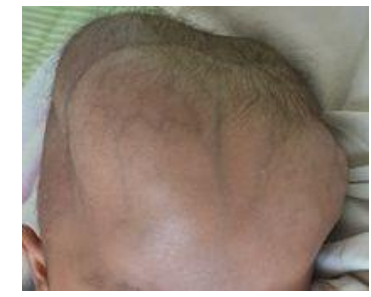

Fig. 6. Increased swelling after chemotherapy.

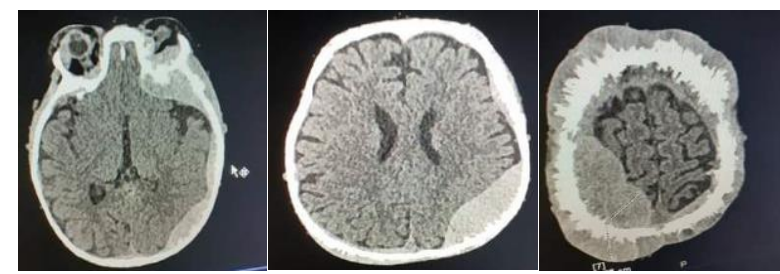

Fig. 7. Post chemotherapy brain scan.

\section{DISCUSSION}

Malignant dural non-Hodgkin's lymphoma is an extremely rare form of non-Hodgkin's lymphoma and its occurrence in children is exceptional. According to the literature, the average age of diagnosis is 50 years [5].

The variability of its clinical manifestations constitutes a diagnostic difficulty responsible for a delay in management. In our case, the first clinical manifestations were multiple swellings of the scalp, exophthalmos, and bone pain in the limbs, which did not alarm the parents for a consultation. According to the literature, the most frequent initial symptoms are scalp swelling, headache, epilepsy, and meningeal irritation [6], which motivate the consultation, and then there may be disturbances in the blood count during the diagnosis.

The most frequent histological type found is large cell B lymphoma with a favorable prognosis and relatively benign [7]. In the reported case, it is a rare non-Hodgkin's malignant lymphoma that is small cell.

Several differential diagnoses must be eliminated in front of a primary lymphoma of the dura mater such as extradural hematoma, meningioma, meningeal sarcomas, meningeal metastases and in front of the swelling or mass at the level of the scalp, it is necessary to take into account the possibility of the occurrence of lymphomas [8].

Regarding the treatment, it depends on several criteria such as the presence or not of a systemic lymphoma, its aggressiveness, and the stage of malignancy [7]. There is no standard protocol for the treatment of dural lymphoma due to the rarity of this condition [9]. Currently, chemotherapy and immunotherapy considerably improve the prognosis of patients. An indication for radiotherapy is very limited but there is a place for it in the localized form of non-Hodgkin's lymphoma with a good therapeutic approach to limit the morbidity and mortality related to the treatment [2]. In developing countries such as Madagascar, polychemotherapy alone is the most commonly used treatment [1]. In the reported case, despite five chemotherapy sessions spaced 21 days apart, no improvement was found. According to the literature, after chemotherapy sessions, a good evolution was found in the majority of cases, considering that lymphomas in children are chemosensitive [1]. 


\section{CONCLUSIONS}

Primary dural non-Hodgkin's malignant lymphomas are rare, especially in children. The diagnosis is suspected clinically, radiologically and anatomopathologically is the only key to confirm it. Their evolution and prognosis are very different and still require efforts for a good management.

\section{REFERENCES}

[1] Randriamampianina T, Rahantamalala MI, Dinafanomezana HR, Rakoto Alson AO. "Profil épidémio-clinique et évolutif des lymphomes malins non hodgkinien vus au service d'oncologie du CHUJRA Antananarivo Madagascar." Journal of Medical care Research and Review [En ligne]. 2019 [Consulté le 20/01/2021]; 2 (10): [04 pages]. Consultable à l'URL: https://core.ac.uk/download/pdf/233889320.pdf.

[2] Drouet F, Cahu X, Pointreau Y, Denis F, Mahé MA. "Lymphomes malins non hodgkiniens." EMC Cancer/Radiothérapie. 2010
Novembre; 14(1): 210-229. DOI: https://doi.org/10.1016/S12783218(10)70025-1.

[3] Bordessoule D. "Objectif ECN : N³16a Lymphomes malins non hodgkiniens." France: Université de Limoges; 2016.

[4] Andon A, Vassal G, Hartmann O, Couanet D, Patte C. "Les lymphomes malins non hodgkiniens." France: Institut GustaveRoussy; 2004 Janvier.

[5] Brito AB, Reis F, de Souza CA, Vassallo J, and Lima CSP "Intracranial primary dural diffuse large B-cell lymphoma successfully treated with chemotherapy." Int J Clin Exp Med. 2014; 7(2): 456-60.

[6] Mohamed T, Bouziane A, Nassar I, Ajana A, Moatassim Billah N. "Exophtalmie bilateral révelatrice d'un lymphoma méningé." EMC Journal of Neuroradiology. 2016 Mars; 43(2): 128.

[7] Gader G, Badri M, Zouaghi M, Ben Salem M, Bahri K, Zammel I. "Le Lymphome fronto-orbitaire: à propos d'un cas et revue de la littérature." EMC Neurochirurgie. 2020 Août; 66(4): 321.

[8] Nair R, Arora N, Mallath MK. "Epidemiology of Non-Hodgkin's Lymphoma in India." International Journal of Cancer Research and Treatment [En ligne]. 2016 Juillet [Consulté le 20/01/2021]; 91 (1): [08 pages]. Consultable à l'URL https://www.karger.com/Article/Abstract/447577.

[9] Iwamoto FM, Abrey LE. "Primary dural lymphomas: a review." Neurosurg Focus. 2006; 21(5): E5. 